\title{
New Additions to Inventory of Marine Mollusc Species from Santa Catarina State/SC, Central Southern Brazil
}

Ignacio Agudo-Padrón A*

Project "Avulsos Malacológicos - AM," Caixa, Florianópolis, Santa Catarina - SC, Brazil

\begin{abstract}
Based on the analysis of bibliographical technical informations of recent production, a new total of 114 forms of marine molluscs (73 Gastropoda, 35 Bivalvia, 5 Scaphopoda and 1 Polyplacophora) is added and incorporated into the systematic malacological inventory of the State of Santa Catarina/ SC, central Southern Brazil, included in 92 genera and 55 families, bringing to 785 species and subspecies registration of regionally known species (12 Polyplacophora, 473 Gastropoda, 15 Scaphopoda, 261 Bivalvia, and 24 Cephalopoda).
\end{abstract}

Keywords: Marine molluscs; Santa Catarina State; Southern Brazil; Species inventory

\section{Introduction}

The coastal plain of the State of Santa Catarina/ SC [1], 255' $41^{\prime \prime} S$ - $29^{\circ} 23^{\prime} 55^{\prime \prime}$, having a length of $580 \mathrm{~km}$ of meandering and diverse mainland and island coast (Figure 1), is located on a large "transition zone", still subject to technical discussions because of the complexity of its nuances and dynamic oceanographic, result of "convergence" between two major ocean currents: "Brazil", hot to the North, and "Falkland or Malvinas", cold to the South, apparently the section of the coast of Brazil between "Rio de Janeiro/ RJ" and "Santa Catarina/ SC" regions functions as an "ecological filter", preventing the dispersal of species, they are thermophilic - Typical of the marine zoogeographical Caribbean Province - or cryophilic - typical of the Patagonian marine zoogeographical Province [2,3].

Sensibly, this condition determines in the local environment a sensitive, diverse and heterogeneous biotic faunal composition, whose characteristics are to have a high potential on the contemporary practice of marine malaco-culture [4] as well as incentive for the development of accurate research on the marine biotic diversity occurring in it $[4,5]$.

Continuing the work of regional survey, the present contribution incorporates in this opportunity the record of further $\mathbf{1 1 4}$ forms to previous systematic inventory of known marine molluscs of the Santa Catarina's State/ SC, the geographical central portion of southern Brazil region [3], included in 92 genera and 55 families, raise the State record for 785 species and subspecies regionally known (12 Polyplacophora, 473 Gastropoda, 15 Scaphopoda, 261 Bivalvia, and 24 Cephalopoda) [6], based on analysis of biogeographical mappings and other relevant informations for 569 regional species contained in the literature of recent production [7] who working in the specific geographical region known as "Argentine Malacological Province - AMP", which includes coasts up to $300 \mathrm{~m}$ depths south of Brazil starting at Santa Catarina State/ SC in Cape/ Lighthouse Santa Marta (28 $36^{\circ} \mathrm{S}-48^{\circ} 48^{\prime} \mathrm{W}$ ) (Figure 1 - Southern sector) to Rio Grande do Sul State/ RS in Chuí ( $33^{\circ} 41^{\prime 2} 27^{\prime \prime S}$ - $53^{\circ} 27^{\prime} 25^{\prime \prime} \mathrm{W}$ ) - a large continuous strip of sands, all of the Oriental Republic of Uruguay and the Argentine Republic up to $42^{\circ} \mathrm{S}$ parallel in the Valdés Península [7], some of them already mentioned in previous short regional compilation [8].

However, to the biogeography of this species, recently Barroso et al. [9] adds the following (sic): "... Considering the results of cluster and ordination analyses, this study proposes to divide the Brazilian coast into four regions ....(3) South-eastern Brazil Area (from southern Bahia to

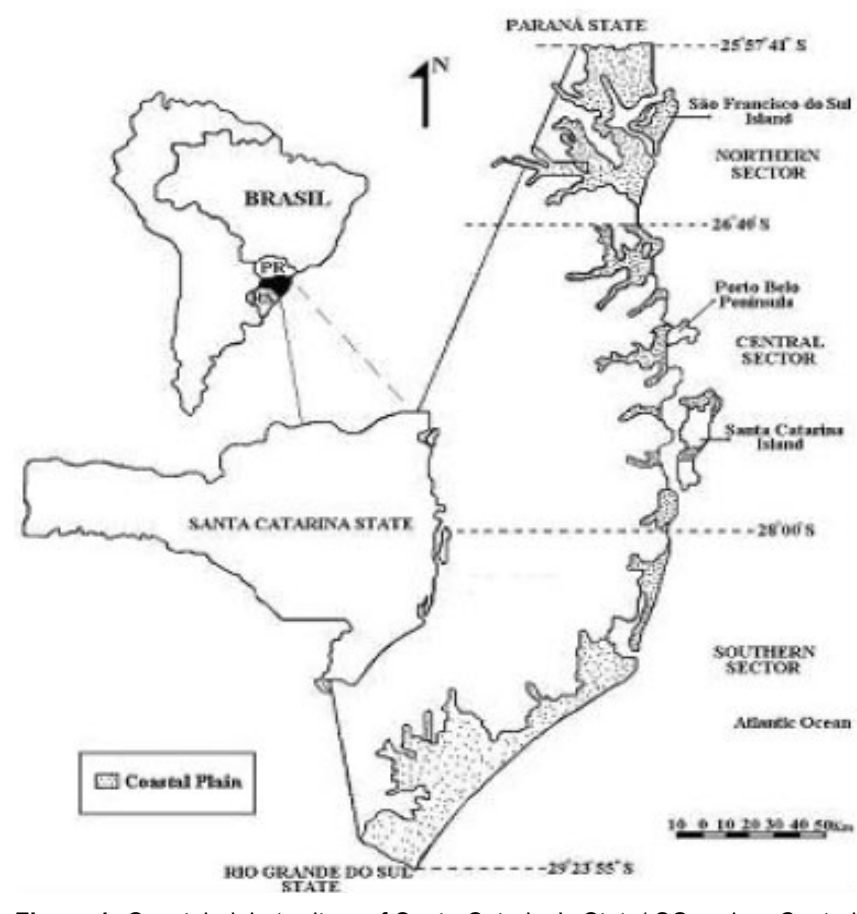

Figure 1: Coastal plain territory of Santa Catarina's State/ SC region, Central Southern Brazil.

Santa Catarina, Brazil, transition zone), and (4) Argentinian Province (Rio Grande do Sul state)...".

Additionally, the recent new species Pugilina tupiniquim Abbate and Simone in 2015 has come to represent the family Melongeniidae in

*Corresponding author: Ignacio Agudo-Padrón A, Project "Avulsos Malacológicos - AM," Caixa Postal (P.O. Box) 010, 88010-970 Centro, Florianópolis, Santa Catarina - SC, Brazil, Tel: (048) 99037948; E-mail: ignacioagudo@gmail.com

Received April 23, 2016; Accepted May 08, 2017; Published May 10, 2017

Citation: Ignacio Agudo-Padrón A (2017) New Additions to Inventory of Marine Mollusc Species from Santa Catarina State/SC, Central Southern Brazil. J Aquac Res Development 8: 478. doi: 10.4172/2155-9546.1000478

Copyright: (c) 2017 Ignacio Agudo-Padrón A. This is an open-access article distributed under the terms of the Creative Commons Attribution License, which permits unrestricted use, distribution, and reproduction in any medium, provided the original author and source are credited. 
Citation: Ignacio Agudo-Padrón A (2017) New Additions to Inventory of Marine Mollusc Species from Santa Catarina State/SC, Central Southern Brazil. J Aquac Res Development 8: 478. doi: 10.4172/2155-9546.1000478

Page 2 of 5

Brazil [10], replacing in Santa Catarina State/ SC the previous specific status Pugilina morio [3].

In general, the taxonomic arrangement presented in this report basically continues accompanying the original proposal contained in Agudo-Padrón [3], based on the monographic contributions of Garcia et al. [11], Rios [12] and Thomé et al. [13] mainly.

\section{Systematic Species Relation/Inventory}

Class Polyplacophora Gray, 1821

Order Neoloricata Bergenhayn, 1955

Family Ischnochitonidae Dall, 1889

Chaetopleura spinulosa (Gray, 1828)

Class Gastropoda Cuvier, 1787

Subclass Prosobranchia Milne Edwards, 1848

Order Archaeogastropoda Thiele, 1925

Family Scissurellidae Gray, 1847

Anatoma aedonia (Watson, 1886)

Family Haliotidae Rafinesque, 1815

Haliotis aurantium Simone, 1998

Family Fissurellidae Fleming, 1822

Emarginula tuberculosa Libassi, 1859

Family Trochidae Rafinesque, 1815

Calliostoma nordenskjoldi Strebel, 1907

Calliostoma moscatelli Quinn, 1992

Calliotropis calatha (Dall, 1927)

Mirachelus clinocnemus Quinn, 1979

Solariella lubrica (Dall, 1881)

Falsimargarita terespina Simone, 2008*

*For an inconvenient omission (error) this species, Falsimargarita terespina Simone, 2008, ended up not being included or counted in the anterior inventory $[3,4]$, although previously reported in the regional literature for the State [14-17].

Family Turbinidae Rafinesque, 1815

Arene microforis (Dall, 1889)

Arene variabilis (Dall, 1889)

Homalopoma boffii Marini, 1975

Family Seguenziidae Verrill, 1884

Ancistrobasis costulata (Watson, 1879)

Order Mesogastropoda Thiele, 1925

Family Caecidae Gray, 1850

Caecum achironum de Folin, 1867

Meioceras nitidum (Stimpson, 1851)

Family Cerithiidae Fleming, 1822
Bittiolum varium (Pfeiffer, 1840)

Litiopa melanostoma Rang, 1829

Alaba incerta (d’Orbigny, 1842)

Family Fossaridae Troschel, 1861

Macromphalina (= Megalomphalus) argentina Castellanos, 1975

Family Ovulidae Fleming, 1822

Pseudosimnia (= Primovula) vanhyningi (M. Smith, 1940)

Family Atlantidae Wiegmann \& Rhute, 1832

Atlanta fusca Souleyet, 1852

Atlanta turriculata d'Orbigny, 1836

Oxygyrus keraudrenii (Lesueur, 1817)

Family Carinariidae Blainville, 1818

Cardiopoda richardi Vayssiêre, 1903

Family Cerithiopsidae Adams \& Adams, 1853

Cerithiopsis fusiformis C.B. Adams, 1845

Family Epitoniidae S.S. Berry, 1910

Epitonium dallianum Verrill \& Smith, 1880

Epitonium celesti (Aradas, 1854)

Cylindriscala andrewsii (Verrill, 1882)

Family Janthinidae Leach, 1823

Janthina globosa (Swainson, 1822)

Family Eulimidae Risso, 1826

Eulima auricincta Abbott, 1859

Eulima bifasciata (d’Orbigny, 1842)

Melanella conoidea (Kurtz \& Stimpson, 1851)

Order Neogastropoda Wenz, 1938

Family Muricidae Rafinesque, 1815

Murexiella hidalgoi (Crosse, 1869)

Orania (= Urosalpinx) fusulus (Brocchi, 1814)

Cytharomorula grayi (Dall, 1889)

Trophon iarae Houart, 1998

Family Olividae Latreille, 1825

Olivella (Olivina) riosi (Klappenbach, 1991)

Family Marginellidae Lamarck, 1799

Eratoidea (= Dentimargo) janeiroensis (E.A. Smith, 1915)

Prunum fulminatum (Kiener, 1841)

Granulina ovuliformis (d’Orbigny, 1841)

Suborder Toxoglossa Troschel, 1848

Family Turridae Swainson, 1840

Splendrillia (= Syntomodrillia) carolinae (Bartsch, 1934) 
Citation: Ignacio Agudo-Padrón A (2017) New Additions to Inventory of Marine Mollusc Species from Santa Catarina State/SC, Central Southern Brazil. J Aquac Res Development 8: 478. doi: 10.4172/2155-9546.1000478

Spirotropis patagonica (d'Orbigny, 1841)

Spirotropis stirophora (Watson, 1881)

Leucosyrinx hemimeres (Watson, 1881)

Benthomangelia macra (Watson, 1881)

Daphnella retifera Dall, 1889

Pleurotomella cala (Watson, 1886)

Order Heterostropha Fisher, 1885

Family Pyramidellidae Gray, 1840

Eulimastoma canaliculatum (C.B. Adams, 1850)

Eulimastoma engonium (Bush, 1885)

Eulimastoma surinamense (Altena, 1975)

Turbonilla farinatiae Pimenta \& Absalão, 2004

Turbonilla zulmae Pimenta \& Absalão, 1998

Careliopsis styliformis Mörch, 1875

Subclass Ophisthobranchia Milne Edwards, 1848

Order Cephalaspidea P. Fischer, 1883

Family Cylichnidae H. \& A. Adams, 1854

Cylichna discus Watson, 1883

Scaphander watsoni Dall, 1881

Volvulella paupercula (Watson, 1883)

Volvulella minuta (= recta) (Bush, 1885)

Order Anaspidea Fischer, 1883

Family Aplysiidae Lamarck, 1809

Stylocheilus longicauda (Quoy \& Gaimard, 1832)

Order Thecosomata Blainville, 1824

Suborder Euthecosomata Neisenheimer, 1905

Family Limacinidae Gray, 1847

Limacina inflata (d'Orbigny, 1836)

Limacina lesueuri (d’Orbigny, 1836)

Limacina bulimoides (d'Orbigny, 1836)

Limacina helicina (Phipps, 1774)

Limacina retroversa (Fleming, 1823)

Family Cavoliniidae Fischer, 1883

Diacavolinia (= Cavolinia) longirostris (Blainville, 1821)

Diacria quadridentata (Lesueur, 1813)

Diacria trispinosa (Blainville, 1821)

Clio antarctica (= andreae) Dall, 1908

Hyalocylis striata (Rang, 1828)

Styliola subula (Quoy \& Gaimard, 1827)

Cuvierina columnella (Rang, 1827)
Family Clionidae Rafinesque, 1815

Clione limacina antarctica (E.A. Smith, 1902)

Order Nudibranchia Blainville, 1814

Family Polyceridae Alder \& Hancock, 1845

Polycera (= Galacera) marplatensis (Franceschi, 1928)

Family Dotoidae H. \& A. Adams, 1854

Doto uva Marcus, 1955

Class Scaphopoda Bonn, 1862

Order Dentaliida da Costa, 1776

Family Dentaliidae Gray, 1834

Paradentalium gouldii (Dall, 1889)

Graptacme calama (Dall, 1889)

Family Gadilinidae Stoliczka, 1868

Episiphon didymum (Watson, 1879)

Order Gadilida Stoliczka, 1868

Suborder Gadilimorpha Steiner, 1992

Family Gadilidae Stoliczka, 1868

Gadila braziliensis (Henderson, 1920)

Gadila pandionis (Verrill \& Smith, 1880)

Class Bivalvia Linnaeus, 1758

Subclass Protobranchia Woodward, 1892

Order Nuculoida Dall, 1889

Superfamily Nuculanoidea Adams \& Adams, 1858

Family Nuculanidae Adams \& Adams, 1858

Propeleda (= Nuculana) fortiana (Esteves, 1984)

Family Malletidae Adams \& Adams, 1858

Malletia subaequalis Sowerby, 1870

Order Arcoidea Lamarck, 1809

Family Limopsidae Dall, 1895

Limopsis davinae Esteves, 1984

Limopsis minuta (Philippi, 1836)

Family Philobryidae Bernard, 1897

Cosa brasiliensis Klappenbach, 1966

Order Mytiloida Ferussac, 1822

Superfamily Mytiloidea Rafinesque, 1815

Family Mytilidae Rafinesque, 1815

Mytilus edulis platensis d'Orbigny, 1846

Brachidontes darwinianus (= exustus) (d'Orbigny, 1846)

Brachidontes rodriguezii (d'Orbigny, 1846)

Amygdalum dendriticum Mühlfeld, 1811 
Citation: Ignacio Agudo-Padrón A (2017) New Additions to Inventory of Marine Mollusc Species from Santa Catarina State/SC, Central Southern Brazil. J Aquac Res Development 8: 478. doi: 10.4172/2155-9546.1000478

Amygdalum saggittatum (Rehder, 1934)

Suborder Pectinina Waller, 1978

Superfamily Pectinoidea Rafinesque, 1815

Family Propeamusiidae Abbott, 1884

Cyclopecten leptaleus (Verrill, 1884)

Propeamussium pourtalesianum (Dall, 1886)

Subclass Heterodonta Neumayr, 1884

Order Veneroida Adams \& Adams, 1856

Family Thyasiridae Dall, 1901

Subfamily Thyasirinae Dall, 1901

Axinulus (= Thyasira) croulinensis (Jeffreys, 1874)

Superfamily Galeommatoidea Gray, 1840

Family Lasaeidae Gray, 1847

Subfamily Lasaeinae Gray, 1847

Lasaea adansoni (Gmelin, 1791)

Kellia suborbicularis (Montagu, 1803)

Superfamily Carditoidea Fleming, 1820

Family Carditidae Fleming, 1820

Subfamily Carditamerinae Chavan, 1969

Cyclocardia moniliata (Dall, 1902)

Pleuromeris sanmartini Klappenbach, 1971

Family Condylocardiidae Bernard, 1897

Warrana (= Americuna) besnardi Klappenbach, 1962

Superfamily Tellinoidea Blainville, 1814

Family Tellinidae Blainville, 1814

Subfamily Tellininae Blainville, 1814

Tellina petitiana d'Orbigny, 1846

Tellina iheringi Dall, 1900

Tellina gibber Ihering, 1907

Family Semelidae Stoliczka, 1870

Abra uruguayensis (Pilsbry, 1897)

Family Psammobiidae Fleming, 1828

Subfamily Psammobiinae Fleming, 1828

Heterodonax bimaculatus (Linnaeus, 1758)

Superfamily Veneroidea Rafinesque, 1815

Family Veneridae Rafinesque, 1815

Subfamily Pitarinae Stewart, 1930

Callista eucymata (Dall, 1889)

Family Petricolidae Deshayes, 1831

Cooperella riosi (= atlantica) Huber, 2010
Order Myoida Lamarck, 1809

Superfamily Myoidea Lamarck, 1809

Family Myidae Lamarck, 1809

Sphenia fragilis (= antillensis) (H. \& A. Adams, 1854)

Family Corbulidae Lamarck, 1818

Subfamily Corbulinae Lamarck, 1818

Corbula patagonica d'Orbigny, 1846

Order Pholadina Adams \& Adams, 1858

Family Teredinidae Rafinesque, 1815

Subfamily Bankiinae Turner, 1966

Bankia fimbriatula Moll \& Roch, 1931

Order Pholadomyoida Newell, 1965

Superfamily Pandoroidea Rafinesque, 1815

Family Periplomatidae Dall, 1895

Periploma compressum d'Orbigny, 1846

Family Lyonsiidae Fischer, 1887

Lyonsia (= Entodesma) alvarezii d'Orbigny, 1846

Superfamily Poromyoidea Dall, 1886

Family Poromyidae Dall, 1844

Poromya cymata Dall, 1889

Family Cuspidariidae Dall, 1886

Plectodon (= Cuspidaria) braziliensis (E.A. Smith, 1915)

Cuspidaria platensis E.A. Smith, 1915

Cardiomya cleryana (d'Orbigny, 1842)

Family Verticordiidae Stoliczka, 1871

Haliris (= Verticordia) fisheriana Dall, 1881

Trigonulina (= Verticordia) ornata d'Orbigny, 1853

\section{References}

1. Lindner A (2014) A vida marinha de Santa Catarina, Vida Marinha de Santa Catarina. Florianópolis: UFSC.

2. Agudo-Padrón Al, Bleicker MS (2014) Acréscimos ao cadastro de moluscos marinhos recentes com ocorrência no Estado de Santa Catarina, região central sul do Brasil. Boletín de la Asociación Argentina de Malacologia 4: 14-15.

3. Agudo-Padrón $\mathrm{Al}$ (2015a) Inventário sistemático revisado y actualizado de lós moluscos marinos ocurrentes em El Estado de Santa Catarina, Brasil. Revista Brasileira de Gestão Ambiental e Sustentabilidade 2: 59-75.

4. Agudo-Padrón $\mathrm{Al}$ (2015b) Mollusc aquaculture and malacological research in Santa Catarina State (Central Southern Brazil region): A brief synthetic critical review. Braz J Biol Sci 2: 377-380.

5. Agudo-Padrón Al (2012) Mollusc fauna in the Atlantic Slope region of the Southern Cone of South America: A preliminary bio-geographical interpretation. Int J Aquac 2: 15-20.

6. Agudo-Padrón Al (2016) The mollusk fauna of Santa Catarina State/ SC, central southern Brazil: A final general balance after two decades of research, with special emphasis on the freshwater species. FMCS Newsletter Ellipsaria 18: 29-32.

7. Forcelli D, Narosky T (2015) Uruguayan seashells - Moluscos marinos de Argentina, Uruguay y Brasil. Buenos Aires: Vázquez Mazzini Editores. 
Citation: Ignacio Agudo-Padrón A (2017) New Additions to Inventory of Marine Mollusc Species from Santa Catarina State/SC, Central Southern Brazil. J Aquac Res Development 8: 478. doi: 10.4172/2155-9546.1000478

8. Viglione GB (2011) Caracoles marinos del Uruguay, Argentina y Brasil. Maldonado.

9. Barroso CX, Lotufo Da TMC, Mattheus-Cascon H (2016) Biogeography of Brazilian prosobranch gastropods and their Atlantic relationships. ¡J Biogeogr 1: 1-12.

10. Abbate D, Simone LRL (2015) Review of Pugilina from the Atlantic, with description of a new species from Brazil (Neogastropoda, Melongenidae). Afr Invertebr 56: 559-577.

11. García FJG, Álvarez MD, Troncoso JS (2008) Opistobranquios de Brasil: Descripción y distribución de Opistobranquios del litoral de Brasil y del Archipiélago Fernando de Noronha. Vigo: Feito.

12. Rios E de C (2009) Compendium of Brazilian Sea Shells. Rio Grande: Evangraf.
13. Thomé JW, Gil G, Bergonci PEA, Tarasconi JC (2010) As conchas das nossas praias. Porto Alegre: Redes Editora.

14. Agudo-Padrón Al, Bleicker MS (2008) Malacofauna marinha catarinense Estado atual do conhecimento dos moluscos marinhos. V: Acréscimos ao cadastro de espécies. Informativo SBMa 39: 8-10.

15. Agudo-Padrón Al, Bleicker MS (2009) Malacofauna marinha catarinense. VII: Aspectos gerais do seu conhecimento. Informativo SBMa 40: 4-11.

16. Agudo-Padrón Al, Bleicker MS (2011) Additional new records on continental and marine molluscs of Santa Catarina State, SC, Southern Brazil region: Syntesis and check list. FMCS Newsletter Ellipsaria 13: 20-26.

17. Agudo-Padrón Al, Bleicker MS, Saalfeld K (2009) Recent marine molluscs of Santa Catarina State, SC, southern Brazil region: A comprehensive synthesis and check list. Visaya Net 24: 1-17. 